\title{
25th Anniversary of European Radiology
}

\author{
Maximilian F. Reiser $^{1}$
}

(C) European Society of Radiology 2016

This year we celebrate the 25th anniversary of European Radiology. We take this occasion as an opportunity to look back on the last two-and-a-half decades, and to reflect on the lessons which can be learned for the future. Radiology has seen breathtaking advances over the past few decades, which have greatly impacted patient and health care in general. With modern radiological methods, precise diagnoses can be obtained without exposing patients to major stress or discomfort. Minimally invasive radiological interventions are effectively replacing many surgical procedures. Basic scientists, engineers and clinical radiologists have dedicated themselves to the development of new methods, assessment of diagnostic effectiveness, and outcome research in order to advance the field of radiology. Numerous publications in European Radiology document these advances; they have expanded our knowledge, provided inspiration for further research and development, and contributed to the improvement of radiological practice for the benefit of our patients.

We selected 25 articles from the last 25 years to evidence these impressive developments. Articles were chosen based on the number of citations the articles received, the different fields of radiology, and the methods used, in order to present a broad and balanced overview. The choice was difficult, as there are many papers which deserved inclusion in this collection. However, there had to be some form of limitation, and selecting 25 articles to represent 25 years of European Radiology seemed like a fair choice.

Maximilian F. Reiser

Maximilian.Reiser@med.uni-muenchen.de

1 Institut für Klinische Radiologie, Klinikum der Universität München, Marchioninistr. 15, 81377 Munich, Germany

\section{The art of writing}

Scientific journals play an important role in disseminating the results of research. The information provided must be sound and unbiased. In addition, an article must be well structured and must satisfy the criteria of good writing. M. Laniado's article, "How to present research data consistently in a scientific paper" [1], published in European Radiology in 1996, outlines in a practical manner the steps an author should follow to get a manuscript accepted.

He starts out by advising the author to slip into the role of the reviewer in order to better understand the reviewer's perspective. Other fundamental considerations in Laniado's article include the following: The introduction should clearly expose the motivation of the research; the material and methods section should follow the sequence of steps in a logical order and allow the reader to reproduce the research. Mentioning institutional review board (IRB) approval and statement of informed consent is indispensable. The structure of the results section should follow the material and methods. The conclusions should avoid overemphasis and should use a modest tone. These recommendations made by Laniado in 1996 are still valid today and can be adopted as they stand.

\section{Contrast media: the never-ending story}

An important change for contrast media has come within the last decades. With high-osmolar ionic contrast media, arteriographies were painful procedures. Therefore, the introduction of non-ionic low-osmolarity iodinated contrast media with low chemotoxicity in the late 1970 s was enthusiastically embraced by both patients and radiologists. Together with digital subtraction angiography (DSA), angiographic procedures became much less cumbersome. In Europe, 
Gmeinwieser and Wenzel-Hora [2] performed a clinical phase II/III trial on peripheral and penile angiography using an isoosmolar contrast agent and found excellent local tolerance, without significant pain or unpleasant heat sensation. These results were confirmed by numerous other studies, and this new class of contrast media also proved favourable in terms of toxic effects on the kidney, heart and brain.

As intra-arterial angiography was increasingly replaced by computed tomography (CT) and magnetic resonance angiography (MRA) as well as vascular ultrasound, iodinated contrast media were increasingly employed on a large scale in contrast-enhanced CT. However, even low-osmolarity contrast agents are not without risk, and can cause contrastinduced nephropathy $(\mathrm{CIN})$. There are many reports in the literature concerning CIN which have come to divergent, if not contradictory, conclusions. To provide radiologists with a reliable source of information, consensus reports on CIN were published by Morcos, Thomsen and Webb in 1999 [3]. These were updated in 2011 by Stacul et al. [4] on behalf of the Contrast Media Safety Committee of the European Society of Urogenital Radiology (ESUR). Based on a systematic review of the literature, the definition of CIN was critically analysed and the risk factors for CIN were revisited. The take-home messages are as follows: (1) CIN risk is lower with intravenous than with intra-arterial iodinated contrast medium; (2) eGFR of $45 \mathrm{ml} / \mathrm{min} / 1.73 \mathrm{~m}^{2}$ is the CIN threshold for intravenous contrast medium; (3) hydration with saline or sodium bicarbonate reduces the incidence of CIN; and (4) patients with eGFR $\geq 60 \mathrm{ml} / \mathrm{min} / 1.73 \mathrm{~m}^{2}$ receiving contrast medium can continue metformin normally.

\section{Computed tomography: from dobbin to racehorse}

CT has witnessed extraordinary advances since its inception in the early 1970s. Spiral (helical) CT and multislice (multidetector) CT (MSCT, MDCT), introduced in 1990 and 1999, respectively, have greatly enhanced the capabilities of this modality. Since then, the number of detector rows has steadily increased and the rotation time has been reduced. These innovations have all contributed to making MSCT an extremely powerful technology.

In 2001, Scaglione et al. published an article in European Radiology on the "role of contrast-enhanced helical CT in the evaluation of acute thoracic aortic injuries after blunt chest trauma" [5]. In a large cohort of 1419 consecutive patients enrolled between 1995 and 2000, the authors identified the following signs indicative of acute thoracic aortic injury: intimal flap, pseudoaneurysm, contour irregularity, lumen abnormality and extravasation of contrast material. With these findings detected, no further diagnostic investigations were required. Thoracic aortic angiography - hitherto the examination of choice - could thus be abandoned. Isolated mediastinal haematoma, however, was considered an indirect sign of acute thoracic aortic injury, and thoracic aortography was required.

The major limitation of single-slice spiral CT was the duration of data acquisition, resulting in limited $z$-axis coverage and thicker collimation. With MSCT, scan time could be reduced and $z$-axis coverage extended, and isotropic volume acquisitions were obtained. In 2002, Remy-Jardin et al. compared single-slice spiral CT and MSCT for CT angiography in patients with suspected pulmonary embolism [6]. Compared to single-slice spiral CT, the frequency of motion artefacts was significantly reduced with MSCT, and more subsegmental arteries were interpretable. In particular, patients with impaired respiratory function benefited from the shorter acquisition time of MSCT.

The evaluation of the heart, especially the coronary arteries, makes high demands on the performance of CT systems. This is even more the case for patients with high heart rates or irregular rhythm. In 2009, Lell et al. published an article on "prospectively ECG-triggered high-pitch spiral acquisition for coronary CT angiography using dual source CT" [7]. They employed $2 \times 1280.6-\mathrm{mm}$ sections and a rotation time of $0.28 \mathrm{~s}$. Data acquisition was prospectively ECG-triggered at $60 \%$ of the R-R interval using a pitch of 3.2 and 3.4, respectively. With this technique, excellent image quality was achieved in almost all coronary artery segments. The mean effective dose was $1.0 \pm 0.3 \mathrm{mSv}$. The authors concluded that prospectively ECG-triggered high-pitch MSCT acquisition provided high and stable image quality at a very low radiation dose.

\section{Towards metabolic and functional imaging}

With spectral imaging, CT has become capable of a more precise characterization of normal and pathological tissues beyond X-ray attenuation quantified in Hounsfield units. For this purpose, vendors have employed various strategies, such as layer detectors, gemstone detectors and dual-energy techniques. In 2007, Johnson et al. reported their initial experience with "material differentiation by dual energy CT" [8]. They showed that iodine and collagen could be differentiated from other tissues with three-material decomposition. Distribution of iodine contrast material could be depicted in the brain, the lung, the liver and the kidneys, with or without the underlying tissue. In angiographies, bone structures could be removed to ease depiction of the vessels. With multispectral imaging, different types of stones in the urinary tract (uric acid, calcium) can be differentiated, and perfusion in the heart and lung can be estimated semi-quantitatively. Furthermore, multispectral $\mathrm{CT}$ has become a valuable method in oncologic imaging.

The high temporal resolution of modern MSCT systems allows for a repetitive acquisition of three-dimensional (3D) data sets. In this way, perfusion imaging following injection of 
intravenous contrast material can be obtained. In brain perfusion, imaging has proved its value for the detection of ischaemia and the selection of appropriate therapy. Frölich et al. published an article in 2014 on "time-resolved assessment of collateral flow using 4D CT angiography in large vessel occlusion stroke" [9].

The leptomeningeal collateral blood flow is crucial for infarct size, response to therapy and outcome of patients with acute ischaemic stroke. Volumetric perfusion CT enables time-resolved four-dimensional CTA of the cerebral vessels. In this study, the collateral flow was best visualized with temporally fused maximum intensity projection (tMIP). The total extent of collateral flow, rather than the velocity of collateral filling, was most predictive for clinical outcome. These results underline the enormous potential of MSCT in the management of stroke.

\section{The predicament: radiation exposure}

The expanding role of MSCT in patient care is associated with a marked increase in radiation exposure for the general population. In the medical community, but also in the general public, concerns were raised that this increase in radiation exposure would result in considerable harm to patients by increasing the risk of radiation-induced cancer (Brenner and Hall, 2007, [10]).

Therefore, extensive efforts were undertaken to reduce radiation exposure in MSCT. In 2002, Jakobs et al. analysed how ECG-controlled tube current modulation affects radiation exposure in patients undergoing retrospectively ECG-gated MSCT of the heart [11]. They found a significant dose reduction of $48 \%$ in males and $45 \%$ in females.

In addressing the need for high temporal resolution, vendors of MSCT machines have trodden different paths. Clearly, this can be achieved by increasing the number of detector rows and rotation speed. One company developed a dualsource CT (DSCT) system, and a first performance evaluation was reported by Flohr et al. in 2006 [12]. The system is equipped with two X-ray tubes and two corresponding detectors mounted onto the rotating gantry, with an angular offset of $90^{\circ}$. A key feature of the DSCT, as pointed out by the authors, is a temporal resolution of $83 \mathrm{~ms}$ when single-segment reconstruction is employed. When examining the heart and the coronary arteries, the authors found considerably increased robustness for the imaging of patients with high heart rates.

The reduction of CT radiation dose is of paramount interest to patients, and considerable efforts have been made to meet this challenge. Iterative reconstruction (IR) techniques are a powerful method for limiting radiation dose. In 2013, Willemink et al. published a two-part article in European Radiology [13, 14], in which the authors described the technical principles of IR and the initial results regarding dose reduction and image quality. An extensive literature search was performed and pertinent articles were analysed. Compared to filtered back-projection (FBP), IR allowed for dose reductions of $23 \%$ to $76 \%$, with similar noise, artefacts, and subjective and objective image quality.

Lu et al. attempted to reduce both the radiation dose and the amount of contrast material in CT pulmonary angiography (CTPA) simultaneously [15]. Low tube voltage (80 kVp) and a high-pitch technique were employed. Sinogramaffirmed IR resulted in reduced image noise and improved image quality. With the combination of these parameters, a contrast material volume of $20 \mathrm{ml}$ was sufficient to maintain the same diagnostic accuracy as that with a standard CTPA protocol, and the effective radiation dose was reduced to less than $1 \mathrm{mSv}$.

Quality assessment and optimization of radiologist performance is another key factor in improving radiological examination, and is an indispensable aspect of radiation protection as well. In 2004, Taylor et al. investigated the effect of radiologist experience in CT colonography on reader performance [16]. The authors found that prior experience in gastrointestinal radiology was a distinct advantage, and noted an unpredictable and variable ability in reporting CT colonography. Training via a database of 50 cases may not be sufficient to attain adequate competence.

\section{Power of attraction: magnetism}

Over the past few decades, magnetic resonance imaging (MRI) has developed at a fast pace and with a constant stream of innovations. The 1.5 Tesla (T) unit became the accepted standard for clinical applications. Faster gradients, ever newer sequence types and multiple-receiver coil systems contributed to excellent performance of MRI. With parallel imaging techniques, examinations could be greatly accelerated. Further progress, however, required increasing the magnetic field strength in order to obtain higher signal yield.

In 2005, Schick et al. published a paper in European Radiology [17] on the technical limits and clinical potential of whole-body MRI. At the time, higher magnetic field strengths had not yet been established. Schick pointed out that higher-field MRI lent itself to susceptibility-dependent imaging, chemical shift-selective imaging and spectroscopic techniques. He noted that major problems existed in abdominal and pelvic imaging, which had to be solved. Fortunately, technical developments have helped overcome these limitations, so that $3 \mathrm{~T}$ MRI can now be applied in all indications of clinical MRI.

Because angiography required arterial puncture, radiologists sought to find less invasive methods for visualization of the vessels. With MRI, time-of-flight (TOF) and phasecontrast (PC) angiography enables depiction of the arterial 
and venous vessels. However, there may be problems due to multi-directional and turbulent flow. In 1997, Bongartz et al. addressed the clinical utility of the then recently introduced contrast-enhanced MR angiography [18]. Combining an ultrafast 3D TOF sequence with the first-pass effect of an extracellular contrast medium was able to generate high-quality angiograms. Moreover, acquisition time was able to be reduced, resulting in a shorter breath-hold period, thus leading to better patient acceptance.

\section{Diffusion imaging — not a diffuse affair}

Diffusion-weighted imaging (DWI) is a highly effective method for the characterization of various pathologies. In stroke diagnosis, DWI has become the established standard of reference. However, DWI has also proved to be highly useful in a multitude of other indications, such as the detection of focal liver lesions.

In 2008, Brügel et al. investigated the differentiation of malignant and benign liver lesions using respiratorytriggered diffusion-weighted single-shot echo-planar MRI [19]. The mean apparent diffusion coefficients (ADC) of haemangioma and cysts differed significantly from those of hepatocellular carcinoma (HCC), metastases and focal nodular hyperplasia (FNH). The authors concluded that malignant liver lesions demonstrate significantly lower ADC values than benign lesions, but that interpretation of ADC should be handled with care, since overlap does exist.

The paradigm of precision medicine incorporates the assessment of prognosis and the selection of appropriate therapy. Radiology can contribute meaningfully towards identifying the type of therapy most appropriate for the individual patient.

In men with prostate cancer, it is important to avoid overtreatment and possibly grave side-effects in clinically insignificant cancer. Active surveillance (AS) has become a widely accepted treatment strategy for individuals with low-risk prostate cancer. In 2015, Kim et al. analysed the role of diffusionweighted MRI for prediction of insignificant prostate cancer in potential candidates for AS [20]. Patients with organconfined disease with a Gleason score $\leq 6$ had an ADC value greater than $0.83 \times 10^{-3} \mathrm{~mm}^{2} / \mathrm{s}$, whereas in more aggressive tumours, the ADC values were significantly lower. The authors concluded that tumour ADC values were effective predictors of insignificant prostate cancer in candidates for AS.

The high value of DWI is also emphasized in the 2012 ESUR prostate MR guidelines published in European Radiology by Barentz and other experts from ESUR [21]. This guideline is based on evidence in the literature and consensus expert opinion. It covers methodological aspects of multiparametric MRI (mpMRI) of the prostate, as well as clinical indications and structured reporting of the results.
mpMRI includes T2-weighted images and at least two functional MRI techniques: DWI, MR spectroscopy (MRS) and dynamic contrast-enhanced MRI (DCE-MRI). DWI enables an estimation of the aggressiveness of prostate cancer, and $\mathrm{ADC}$ values correlate with Gleason scores. The guideline proposes the use of the Prostate Imaging Reporting and Data System (PI-RADS) classification for reporting and communicating the results of mpMRI.

\section{Molecular imaging}

The pharmacokinetics of extracellular iodinated X-ray and extracellular gadolinium-based MRI contrast media behave very similarly. For MRI, more specific contrast media have been developed. Hepatotropic gadolinium-based substances, small iron oxide particles and blood-pool contrast media paved the way for molecular imaging. In 2010, Kogita et al. used the hepatotropic agent Gd-EOB-DTPA to investigate the enhancement patterns of hepatocellular carcinoma (HCC) and dysplastic nodules (DNs) [22]. They also assessed the portal blood flow using CT during arterial portography and found that post-contrast EOB ratios decreased significantly as the differentiation in DNs declined. The decrease in portal blood flow was not as pronounced as the decrease in EOB uptake. The authors concluded that a reduction in uptake of Gd-EOBDTPA occurred at an early stage of hepatocarcinogenesis, preceding a reduction in portal blood flow.

These results underscore the great potential of organ- and receptor-specific MRI contrast media. The arduous bureaucratic hurdles and high cost of approval of new contrast media, however, prevent companies from pursuing research in new and more specific contrast media.

With MRI, diagnostic information beyond morphological imaging can be obtained, such as MRS, functional MRI of the brain, DWI and perfusion imaging. In 2007, Padhani et al. examined oxygenation of human tumours, which affects the susceptibility of malignant tumours to radiation therapy and cytotoxic chemotherapy [23]. With blood oxygen leveldependent (BOLD)-MRI, hypoxia within tumours could be assessed. The authors concluded that hypoxia imaging may allow for higher precision in the selection of therapy.

In the age of molecular medicine, biomedical imaging strives for the non-invasive mapping of molecular events in the tissue. Fluorescent probes in the near-infrared spectrum allow for penetration of several centimetres into the tissue. The article in 2003 by Ntziachristos et al., "Fluorescence imaging with near-infrared light: new technical advances that enable in vivo molecular imaging", described the newly developed method of fluorescence-mediated molecular tomography [24], which allowed for 3D imaging of gene expression by resolving fluorescence in deep tissues. 


\section{The rude awakening: NSF}

In contrast to iodine X-ray contrast agents, gadolinium-based contrast media were considered harmless in patients with reduced renal function. Contrast-enhanced MRA was frequently used with increased doses of contrast media, especially when multiple vascular territories had to be examined.

In a first report by Grobner in 2006, however, it became evident that skin lesions could occur in patients with end-stage renal disease who were exposed to gadolinium-based contrast media, a condition referred to as nephrogenic systemic fibrosis (NSF) [25]. The molecular structure of these contrast media was found to have a major impact on the risk of NSF. In 2007, ESUR published guidelines on NSF (Thomsen et al., 2007 [26]), which were then updated in 2013 (Thomsen et al. [27]), and the current literature was taken into consideration. The key points of this article can be summarized as follows: patients with a glomerular filtration rate (GFR) below $30 \mathrm{ml} /$ $\min / 1.73 \mathrm{~m}^{2}$ are at increased risk of developing NSF; lowstability gadolinium contrast media show the strongest association with NSF; and adhering to the guidelines regarding gadolinium contrast agents minimizes the risk of NSF. The NSF tragedy should alert the radiological community that we must take warnings seriously and that unexpected and previously unknown side effects may occur. On the other hand, a critical and evidence-based approach is required.

\section{The sound of silence}

European Radiology receives numerous submissions in the field of ultrasound, and highly interesting articles have been published. Recently, elastography and contrast-enhanced ultrasound have attracted much attention. In 1999, Derchi et al. were among the first to investigate the potential of ultrasound contrast media for improving diagnostic accuracy [28]. Microbubble-based ultrasound contrast media are pure blood-pool agents with which functional evaluation of different organs can be performed.

\section{The health care system under pressure}

The health care system is under high economic pressure, and cost-effectiveness evaluations are of great importance. When PET-CT hybrid systems became available, the question emerged as to whether the higher cost of the hybrid systems was justified by the improvement in diagnostic accuracy. Already in 2000, von Schulthess presented cost calculations in which he compared the pros and cons of hybrid systems and separate modalities [29]. For a brief period after that, only combined PET-CT systems were manufactured by the industry, and proved to be valuable for many diagnostic problems.

\section{Conclusions}

Reading articles from two-and-a-half decades of European Radiology feels a bit like a journey through time. Radiology has been confronted with many challenges, some of which could be overcome through methodological and technical innovations. Others were brought closer to a solution, whereas some remain unchanged and are still topical or have become even more urgent today.

Like other areas of medicine, radiology and associated disciplines strive to implement the ideas and demands of precision medicine and to contribute in a meaningful way. The medical condition of each patient should be characterized as precisely as possible to allow for personally customized treatment. With the help of radiomics, we are able to define a patient with regard to his/her prognosis and the ideal individualized therapy.

Not least due to the demographic trend of a growing number of older patients and the rising prevalence of obesity and metabolic syndrome, the rational and evidence-based use of contrast media has become increasingly important. Given the growing application of imaging techniques that employ ionizing radiation, measures for curbing radiation exposure are indispensable. While we have already made impressive advances in this area, we must remain vigilant.

Interventional radiology contributes greatly to the capability for gentle, less invasive, more cost-effective treatment. We need to document these advantages with multicentre outcome studies in large patient collectives. Similarly, radiological diagnostics must demonstrate that it can improve patient care with cost-effective methods, thus ultimately helping to curb costs in the health care system.

In closing, I find that radiology has made great strides in the last 25 years, and that these advances have been accompanied, furthered and promoted by European Radiology. We aim to continue in this way in the future and hope you will be with us.

Maximilian F. Reiser

Editor-in-Chief

\section{References}

1. Laniado M (1996) How to present research data consistently in a scientific paper. Eur Radiol 6:S16-S18

2. Gmeinwieser JK, Wenzel-Hora BI (1995) Peripheral and penile angiography with iotrolan 280 versus non-ionic monomers: results of the European clinical phase II and III trials. Eur Radiol 5:S30-S38

3. Morcos SK, Thomsen HS, Webb JAW (1999) Contrast mediainduced nephrotoxicity: a consensus report. Eur Radiol 9:1602-1613

4. Stacul F, van der Molen AJ, Reimer P et al (2011) Contrast induced nephropathy: updated ESUR Contrast Media Safety Committee guidelines. Eur Radiol 21:2527-2541 
5. Scaglione M, Pinto A, Pinto F, Romano L, Ragozzino A, Grassi R (2001) Role of contrast-enhanced helical CT in the evaluation of acute thoracic aortic injuries after blunt chest trauma. Eur Radiol 11: 2444-2448

6. Remy-Jardin M, Tillie-Leblond I, Szapiro D et al (2002) CT angiography of pulmonary embolism in patients with underlying respiratory disease: impact of multislice CT on image quality and negative predictive value. Eur Radiol 12:1971-1978

7. Lell M, Marwan M, Schepis T et al (2009) Prospectively ECGtriggered high-pitch spiral acquisition for coronary $\mathrm{CT}$ angiography using dual source CT: technique and initial experience. Eur Radiol 19:2576-2583

8. Johnson TR, Krauss B, Sedlmair M et al (2007) Material differentiation by dual energy CT: initial experience. Eur Radiol 17:15101517

9. Frölich AM, Wolff SL, Psychogios MN et al (2014) Time-resolved assessment of collateral flow using 4D CT angiography in largevessel occlusion stroke. Eur Radiol 24:390-396

10. Brenner DJ, Hall EJ (2007) Computed tomography-an increasing source of radiation exposure. N Engl J Med 357:2277-2284

11. Jakobs TF, Becker CR, Ohnesorge B et al (2002) Multislice helical $\mathrm{CT}$ of the heart with retrospective ECG gating: reduction of radiation exposure by ECG-controlled tube current modulation. Eur Radiol 12:1081-1086

12. Flohr TG, McCollough $\mathrm{CH}$, Bruder $\mathrm{H}$ et al (2006) First performance evaluation of a dual-source CT (DSCT) system. Eur Radiol 16:256-268

13. Willemink MJ, de Jong PA, Leiner T et al (2013) Iterative reconstruction techniques for computed tomography Part 1: technical principles. Eur Radiol 23:1623-1631

14. Willemink MJ, Leiner T, de Jong PA et al (2013) Iterative reconstruction techniques for computed tomography part 2: initial results in dose reduction and image quality. Eur Radiol 23:1632-1642

15. Lu GM, Luo S, Meinel FG et al (2014) High-pitch computed tomography pulmonary angiography with iterative reconstruction at $80 \mathrm{kVp}$ and $20 \mathrm{~mL}$ contrast agent volume. Eur Radiol 24:32603268

16. Taylor SA, Halligan S, Burling D et al (2004) CT colonography: effect of experience and training on reader performance. Eur Radiol 14:1025-1033
17. Schick F (2005) Whole-body MRI at high field: technical limits and clinical potential. Eur Radiol 15:946-959

18. Bongartz GM, Boos M, Winter K, Ott H, Scheffler K, Steinbrich W (1997) Clinical utility of contrast-enhanced MR angiography. Eur Radiol 7:178-186

19. Bruegel M, Holzapfel K, Gaa J et al (2008) Characterization of focal liver lesions by ADC measurements using a respiratory triggered diffusion-weighted single-shot echo-planar MR imaging technique. Eur Radiol 18:477-485

20. Kim JW, Lee CH, Yoo KH et al (2015) Diffusion-weighted magnetic resonance imaging for prediction of insignificant prostate cancer in potential candidates for active surveillance. Eur Radiol 25: 1786-1792

21. Barentsz JO, Richenberg J, Clements R et al (2012) ESUR prostate MR guidelines 2012. Eur Radiol 22:746-757

22. Kogita S, Imai Y, Okada M et al (2010) Gd-EOB-DTPA-enhanced magnetic resonance images of hepatocellular carcinoma: correlation with histological grading and portal blood flow. Eur Radiol 20:2405-2413

23. Padhani AR, Krohn KA, Lewis JS, Alber M (2007) Imaging oxygenation of human tumours. Eur Radiol 17:861-872

24. Ntziachristos V, Bremer C, Weissleder R (2003) Fluorescence imaging with near-infrared light: new technological advances that enable in vivo molecular imaging. Eur Radiol 13:195-208

25. Grobner T (2006) Gadolinium: a specific trigger for the development of nephrogenic fibrosing dermopathy and nephrogenic systemic fibrosis. Nephrol Dial Transplant 21:1104-1108

26. Thomsen HS (2007) ESUR guideline: gadolinium-based contrast media and nephrogenic systemic fibrosis. Eur Radiol 17:26922696

27. Thomsen HS, Morcos SK, Almén T et al (2013) Nephrogenic systemic fibrosis and gadolinium-based contrast media: updated ESUR Contrast Medium Safety Committee guidelines. Eur Radiol 23: 307-318

28. Derchi LE, Martinoli C, Pretolesi F, Crespi G, Buccicardi D (1999) Quantitative analysis of contrast enhancement. Eur Radiol 9:S372S376

29. von Schulthess GK (2000) Cost considerations regarding an integrated CT-PET system. Eur Radiol 10:S377-S380 